\title{
Tracking Air-to-Air Missile Using Proportional Navigation Model with Genetic Algorithm Particle Filter
}

\author{
Hongqiang Liu, Lei Yu, Chenwei Ruan, and Zhongliang Zhou \\ Aeronautics and Astronautics Engineering College, Air Force Engineering University, Xi'an 710038, China \\ Correspondence should be addressed to Hongqiang Liu; lhqkgdsa@yahoo.com
}

Received 17 November 2015; Accepted 25 February 2016

Academic Editor: Vladimir Turetsky

Copyright ( 2016 Hongqiang Liu et al. This is an open access article distributed under the Creative Commons Attribution License, which permits unrestricted use, distribution, and reproduction in any medium, provided the original work is properly cited.

\begin{abstract}
The purpose of this paper is to track the air-to-air missile. Here we put forward the PN-GAPF (Proportional Navigation motion model and Genetic Algorithm Particle Filter) method to solve the problem. The main jobs we have done can be listed as follows: firstly, we establish the missile state space model named as the Proportional Navigation (PN) motion model to simulate the real motion of the air-to-air missile; secondly, the PN-EKF and PN-PF methods are proposed to track the missile, through combining PN motion model with EKF and PF; thirdly, in order to solve the particle degeneracy and diversity loss, we introduce the intercross and variation in GA to the particles resampling step and then the PN-GAPF method is put forward. The simulation results show that the PN motion model is better than the CV and CA motion models for tracking the air-to-air missile and that the PN-GAPF method is more efficient than the PN-EKF and PN-PF.
\end{abstract}

\section{Introduction}

The air-to-air missile is the main weapon in the air combat. The aircraft been chased should maneuver to avoid the attacking missile, after the air-to-air missile was fired by an opposition fighter. The aircraft guidance method for maneuver evasion is based on knowing the missile location and the real-time track [1]. However, we cannot acquire the exact location information of the missile because the measurement for the missile location has great error. We need an on-line filter method to eliminate the error and track the air-to-air missile. How to effectively track the missile is the research content in this paper.

This problem is the domain of the single target tracking. The common target motion models such as the CV, CA, and Current Statistical (CS) models cannot well and truly describe the air-to-air missile maneuver because the missile has a good maneuverability and a supersonic speed [2]. However, we can research from the navigation law point to establish a smarter motion model because the missile maneuver obeys some navigation law [3]. The Proportional Navigation (PN) law is the most common in the air-to-air missiles [4]. Therefore we establish a new PN motion model in 3d Cartesian coordinate system by analysis of the $\mathrm{PN}$ mechanism.
The state space model for tracking the air-to-air missile is obtained further, through combining with the nonlinear measurement equation in the radar spherical coordinate system. The standard Kalman Filter (KF) cannot be used to track the missile because of the nonlinear measurement equation. The Extended Kalman Filter (EKF) and Unscented Kalman Filter (UKF) are used to solve the nonlinear problem, but the methods are not efficient enough in practice [5]. Recently, the Particle Filter (PF) or Monte Carlo (MC) method is widely applied in the nonlinear and non-Gaussian filter problem because $\mathrm{PF}$ is to be able to represent the required unknown probability density function by a scatter of particles sampled from a known probability density function [6]. However, the resampling of standard PF has a disadvantage of the loss of particle diversity [7]. We are inspired by the evolution idea of Genetic Algorithm (GA) to improve the performance of the $\mathrm{PF}$ at resampling step [8]. We take the particles and weights in the PF as the chromosomes and adaptability in the GA, respectively. Then the intercross and variation steps in the GA can be adopted to deal with the particles in resampling step for avoiding the particle degeneracy and loss of diversity. In this paper, Genetic Algorithm Particle Filter (GAPF) combined with the PN motion model (PN-GAPF) is used to track the air-to-air missile. PN-GAPF has better the estimate 
accuracy and the tracking stability, in comparison with other algorithms and models in computer simulation.

This paper is divided into five sections. Section 1 is introduction. Section 2 presents the state space model for tracking the air-to-air missile and the method of establishing the PN motion model. Section 3 describes the nonlinear filter algorithms such as the EKF, PF, and GAPF proposed. Section 4 discusses the performance of the PN motion model and GAPF algorithm on the base of the simulation results. Section 5 summarizes the main research content.

\section{Problem Formulation}

2.1. The State Space Model. The state space model for tracking the air-to-air missile consists of the missile state equation and the measurement equation. In order to simplify the problem, the missile state equation is usually modeled linearly in Cartesian coordinate system and the measurement equation is expressed nonlinearly in spherical coordinate system because of the air-to-air missile measurements given in the radar spherical coordinate system [9].

2.1.1. The Linear Missile State Equation. In general, we estimate the state of the moving air-to-air missile with the discrete-time linear state space in $3 \mathrm{~d}$ Cartesian coordinate system [10]:

$$
\mathbf{X}(k+1)=\mathbf{A X}(k)+\mathbf{v}(k),
$$

where $\mathbf{X}(k+1)$ and $\mathbf{X}(k)$ are the state vector at time $t=$ $(k+1) T$ and $t=k T$ respectively and $T$ is a sampling period. $\mathbf{A}$ is the state transition matrix. $\mathbf{v}(k)$ is the process noise which is modeled as a zero-mean white Gaussian process with covariance matrix $\mathbf{Q}(k)$.

The CV model and the CA model are the most common motion models for tracking air-to-air missile [11]. The state vector $\mathbf{X}_{\mathrm{CV}}(k)$ and the state transition matrix $\mathbf{A}_{\mathrm{CV}}$ of $\mathrm{CV}$ model in $3 \mathrm{~d}$ Cartesian coordinate system are

$$
\begin{aligned}
\mathbf{X}_{\mathrm{CV}}(k)= & {\left[x(k), v_{x}(k), y(k), v_{y}(k), z(k), v_{z}(k)\right], } \\
\mathbf{A}_{\mathrm{CV}} & =\left[\begin{array}{cccccc}
1 & T & 0 & 0 & 0 & 0 \\
0 & 1 & 0 & 0 & 0 & 0 \\
0 & 0 & 1 & T & 0 & 0 \\
0 & 0 & 0 & 1 & 0 & 0 \\
0 & 0 & 0 & 0 & 1 & T \\
0 & 0 & 0 & 0 & 0 & 1
\end{array}\right] .
\end{aligned}
$$

And the state vector $\mathbf{X}_{\mathrm{CA}}(k)$ and the state transition matrix $\mathbf{A}_{\mathrm{CA}}$ of CA model are

$$
\begin{aligned}
& \mathbf{X}_{\mathrm{CA}}(k)=\left[x(k), v_{x}(k), a_{x}(k), y(k), v_{y}(k), a_{y}(k), z(k),\right. \\
& \left.v_{z}(k), a_{z}(k)\right],
\end{aligned}
$$

$$
\begin{aligned}
& \mathbf{A}_{\mathrm{CA}}=\left[\begin{array}{ccc}
\mathbf{E}_{3 \times 3} & \operatorname{dig}(T)_{3 \times 3} & \operatorname{dig}\left(\frac{1}{2} \cdot T^{2}\right)_{3 \times 3} \\
\mathbf{0}_{3 \times 3} & \mathbf{E}_{3 \times 3} & \operatorname{dig}(T)_{3 \times 3} \\
\mathbf{0}_{3 \times 3} & \mathbf{0}_{3 \times 3} & \mathbf{E}_{3 \times 3}
\end{array}\right], \\
& \operatorname{dig}(T)_{3 \times 3}=\left[\begin{array}{ccc}
T & 0 & 0 \\
0 & T & 0 \\
0 & 0 & T
\end{array}\right], \\
& \operatorname{dig}\left(\frac{T^{2}}{2}\right)_{3 \times 3}=\left[\begin{array}{ccc}
\frac{T^{2}}{2} & 0 & 0 \\
0 & \frac{T^{2}}{2} & 0 \\
0 & 0 & \frac{T^{2}}{2}
\end{array}\right]
\end{aligned}
$$

where $x(k), y(k)$, and $z(k)$ are the missile positions along each axis of coordinates. Correspondingly, $v_{x}(k), v_{y}(k)$, and $v_{z}(k)$ are the velocities. $a_{x}(k), a_{y}(k)$, and $a_{z}(k)$ are the accelerators. If sampling the measurement in a very short time, we can consider the missiles maneuvering as an approximate $\mathrm{CV}$ model or CA model [11].

2.1.2. The Nonlinear Measurement Equation. In the real air combat, the measurement of air-to-air missile is as follows in the radar spherical coordinate system [12]:

$$
\mathbf{Z}(k)=[r(k), \theta(k), \varphi(k)],
$$

where $\mathbf{Z}(k)$ is the measurement vector. $r(k)$ is the distance between the aircraft attacked and the air-to-air missile which can be detected by the airborne laser range finder. $\theta(k)$ and $\varphi(k)$ are the pitching and azimuth angles, respectively, which can be detected by the aircraft radar warning device [13].

The nonlinear measurement equation is

$$
\mathbf{Z}(k)=\mathbf{h}(\mathbf{X}(k))+\mathbf{w}(k),
$$

where $\mathbf{h}(\cdot)$ is the nonlinear measurement function which will be given at the next section. $\mathbf{w}(k)$ is the measurement error which is zero-mean white Gaussian noise with covariance matrix $\mathbf{R}(k)$ [14].

2.2. The Proportional Navigation Motion Model. If the air-toair missile movement is modeled just simply as the CV or CA motion model, it will produce great errors of the missile state estimation [4]. It is known that the air-to-air missile is designed to maneuver by the Proportional Navigation law. Therefore, the motion model based on the Proportional Navigation law is a smarter model to reflect the states in the attack process. We describe the relative movement between the aircraft attacked and the air-to-air missile in Figure 1.

2.2.1. The Geographic Coordinate System. We take "northsky-west" geographic coordinate system [15] as the 3d Cartesian coordinate system to describe the relative movement in Figure 1. The origin of coordinates " $O$ " is set at the firing point of the air-to-air missile. The " $O X$ " axis is tangent with longitude and points to the north. The "OY" axis points to the sky and the "OZ" axis points to the west. 


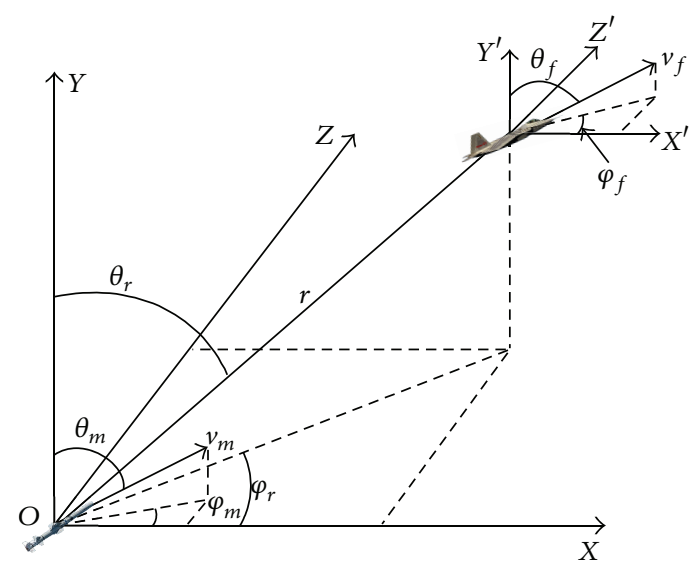

FIGURE 1: The relative movement and variables.

2.2.2. The Relative Movement. We assume that the attacked aircraft position and the missile position are $\left(x_{f}, y_{f}, z_{f}\right)$ and $\left(x_{m}, y_{m}, z_{m}\right)$ in "OXYZ" coordinate system, respectively. $\left(r, \theta_{r}, \varphi_{r}\right)$ are the relative distance and the relative pitching and relative azimuth angles between them in the spherical coordinate system. The relationship of those variables is [16]

$$
\begin{aligned}
r & =\sqrt{\left(x_{f}-x_{m}\right)^{2}+\left(y_{f}-y_{m}\right)^{2}+\left(z_{f}-z_{m}\right)^{2}}, \\
\varphi_{r} & =\arctan \left(\frac{z_{f}-z_{m}}{x_{f}-x_{m}}\right), \\
\theta_{r} & =\arctan \left(\frac{y_{f}-y_{m}}{\sqrt{\left(x_{f}-x_{m}\right)^{2}+\left(z_{f}-z_{m}\right)^{2}}}\right) .
\end{aligned}
$$

The velocity vectors of the aircraft and the missile are $\left(v_{f x}, v_{f y}, v_{f z}\right)$ and $\left(v_{m x}, v_{m y}, v_{m z}\right)$ in the "OXYZ" coordinate system; then $\left(v_{m}, \theta_{m}, \varphi_{m}\right)$ and $\left(v_{f}, \theta_{f}, \varphi_{f}\right)$ are in the spherical coordinate system. The relationships of those variables are

$$
\begin{aligned}
& \frac{d x_{m}}{d t}=v_{m x}=v_{m} \sin \theta_{m} \cos \varphi_{m}, \\
& \frac{d y_{m}}{d t}=v_{m y}=v_{m} \cos \theta_{m}, \\
& \frac{d z_{m}}{d t}=v_{m z}=v_{m} \sin \theta_{m} \sin \varphi_{m}, \\
& \frac{d x_{f}}{d t}=v_{f x}=v_{f} \sin \theta_{f} \cos \varphi_{f}, \\
& \frac{d y_{f}}{d t}=v_{f y}=v_{f} \cos \theta_{f}, \\
& \frac{d z_{f}}{d t}=v_{f z}=v_{f} \sin \theta_{f} \sin \varphi_{f} .
\end{aligned}
$$

Popularly, the Proportional Navigation law can be decoupled in the "OXY" plane and "OXZ" plane [17]:

$$
\begin{aligned}
& \dot{\theta}_{m}=K_{\theta} \dot{\theta}_{r}, \\
& \dot{\varphi}_{m}=K_{\varphi} \dot{\varphi}_{r},
\end{aligned}
$$

where $K_{\theta}$ and $K_{\varphi}$ are the Proportional Navigation coefficients, $2 \leq K \leq 6 . \dot{\theta}_{m} \dot{\varphi}_{m} \dot{\theta}_{r}$ and $\dot{\varphi}_{r}$ are the derivations of $\theta_{m} \varphi_{m} \theta_{r}$ and $\varphi_{r}$, respectively.

\subsubsection{Modeling the Proportional Navigation State Space Model.} We assume that the velocity $v_{m}$ is a fixed value [18] because the roll booster of air-to-air missile works in a short time. Then we take derivative of (7):

$$
\begin{aligned}
& \dot{v}_{m x}=\dot{\theta}_{m} v_{m} \cos \theta_{m} \cos \varphi_{m}-\dot{\varphi}_{m} v_{m} \sin \theta_{m} \sin \varphi_{m}, \\
& \dot{v}_{m y}=\dot{\theta}_{m} v_{m} \sin \theta_{m}, \\
& \dot{v}_{m z}=-\dot{\theta}_{m} v_{m} \cos \theta_{m} \sin \varphi_{m}+\dot{\varphi}_{m} v_{m} \sin \theta_{m} \cos \varphi_{m} .
\end{aligned}
$$

Combining (7) with (8), (10) will be changed to

$$
\begin{aligned}
& \dot{v}_{m x}=v_{m y} K_{\theta} \dot{\theta}_{r} \cos \varphi_{m}-v_{m z} K_{\varphi} \dot{\varphi}_{r}, \\
& \dot{v}_{m y}=v_{m y} K_{\theta} \dot{\theta}_{r} \tan \theta_{m}, \\
& \dot{v}_{m z}=v_{m x} K_{\varphi} \dot{\varphi}_{r}+v_{m y} K_{\theta} \dot{\theta}_{r} \sin \varphi_{m} .
\end{aligned}
$$

Now we take the missile state vector as $\mathbf{X}_{\mathrm{PN}}=\left[x_{m}, v_{m x}\right.$, $\left.y_{m}, v_{m x}, z_{m}, v_{m z}\right]$. According to (11), the continuous-time linear state equation [19] can be established as

$$
\begin{aligned}
\dot{\mathbf{X}} & =\mathbf{F}_{\mathrm{PN}} \mathbf{X}+\mathbf{v} \\
\mathbf{F}_{\mathrm{PN}} & =\left[\begin{array}{cccccc}
0 & 1 & 0 & 0 & 0 & 0 \\
0 & 0 & 0 & K_{\theta} \dot{\theta}_{r} \cos \varphi_{m} & 0 & K_{\varphi} \dot{\varphi}_{r} \\
0 & 0 & 0 & 1 & 0 & 0 \\
0 & 0 & 0 & K_{\theta} \dot{\theta}_{r} \tan \theta_{m} & 0 & 0 \\
0 & 0 & 0 & 0 & 0 & 1 \\
0 & K_{\varphi} \dot{\varphi}_{r} & 0 & K_{\theta} \dot{\theta}_{r} \sin \varphi_{m} & 0 & 0
\end{array}\right] .
\end{aligned}
$$

Then we convert (12) to the discrete linear state equation

$$
\begin{aligned}
\mathbf{X}(k+1) & =\mathbf{A}_{\mathrm{PN}} \mathbf{X}(k)+\mathbf{v}(k), \\
\mathbf{A}_{\mathrm{PN}} & =e^{\mathbf{F}_{\mathrm{PN}} T} .
\end{aligned}
$$


The precise analytic expression of $\mathbf{A}_{\mathrm{PN}}$ cannot be acquired, but we use (15) to get an approximation by taking $k=3[20]$ :

$$
\begin{aligned}
& e^{\mathbf{F}_{\mathrm{PN}} T}=\sum_{k=0}^{\infty} \frac{\left(\mathbf{F}_{\mathrm{PN}} T\right)^{k}}{k !} \approx I+\mathbf{F}_{\mathrm{PN}} T+\frac{\mathbf{F}_{\mathrm{PN}}{ }^{2} T^{2}}{2 !}+\frac{\mathbf{F}_{\mathrm{PN}}{ }^{3} T^{3}}{3 !}
\end{aligned}
$$

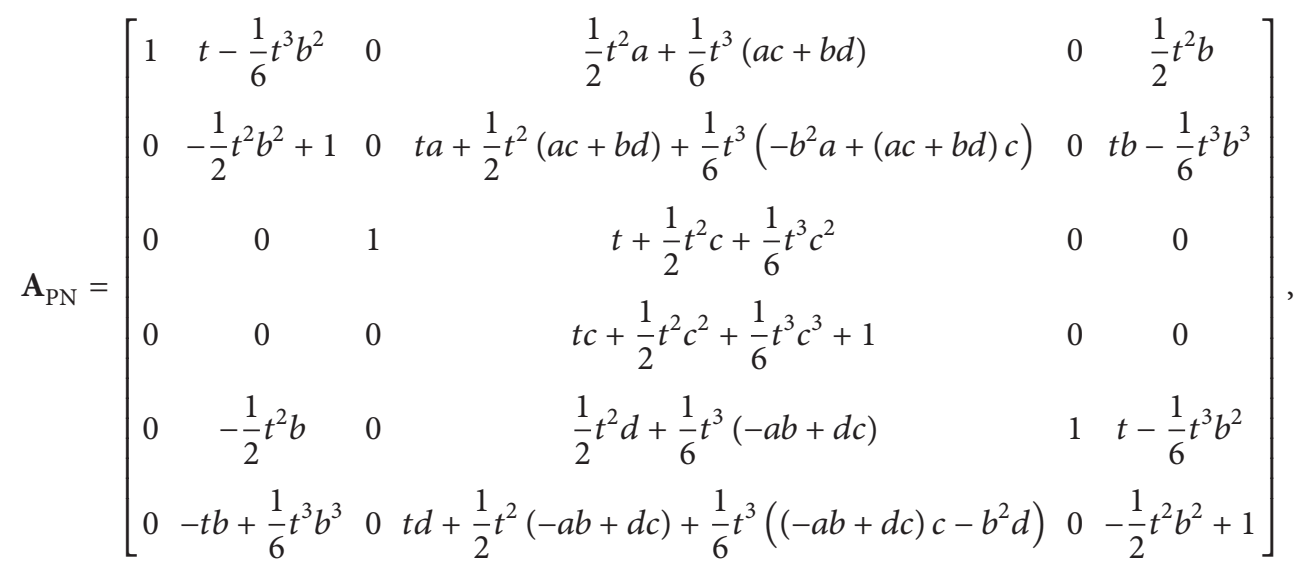

$$
\begin{aligned}
& a=-K_{\theta} \dot{\theta}_{r} \cos \varphi_{m}, \\
& b=-K_{\varphi} \dot{\varphi}_{r}, \\
& c=-K_{\theta} \dot{\theta}_{r} \tan \theta_{m}, \\
& d=K_{\theta} \dot{\theta}_{r} \sin \varphi_{r}, \\
& t=T \text {. }
\end{aligned}
$$

The measurement equation can directly be modeled in discrete form like (5) because the measurements of the missile are detected discretely. According to (6), $\mathbf{h}(\mathbf{X}(k))$ is?

$$
\mathbf{h}(\mathbf{X}(k))=\left[\begin{array}{c}
\sqrt{\left(x_{f}(k)-x_{m}(k)\right)^{2}+\left(y_{f}(k)-y_{m}(k)\right)^{2}+\left(z_{f}(k)-z_{m}(k)\right)^{2}} \\
\arctan \left(\frac{y_{f}(k)-y_{m}(k)}{\sqrt{\left(x_{f}(k)-x_{m}(k)\right)^{2}+\left(z_{f}(k)-z_{m}(k)\right)^{2}}}\right) \\
\arctan \left(\frac{z_{f}(k)-z_{m}(k)}{x_{f}(k)-x_{m}(k)}\right)
\end{array}\right] .
$$

\section{Nonlinear Filtering Algorithm}

The Kalman filter assumptions do not hold because the measurement equation is a nonlinear function in the measurement equation [21]. Therefore, for the tracking missile, there are three approximate nonlinear filters to solve the nonlinear problem in the paper.

3.1. The Extended Kalman Filter with the PN Motion Model. The EKF approximates the nonlinear function through utilizing the first term in a Taylor expansion of the nonlinear function [22]: 


$$
\begin{aligned}
& \mathbf{H}_{k}=\left.\frac{d \mathbf{h}(\mathbf{X})}{d \mathbf{X}}\right|_{\mathbf{X}=\mathbf{X}(k \mid k-1)}, \\
& \mathbf{H}_{k}=\left[\begin{array}{llllll}
\frac{\partial r}{\partial x_{m}} & \frac{\partial r}{\partial v_{m x}} & \frac{\partial r}{\partial y_{m}} & \frac{\partial r}{\partial v_{m y}} & \frac{\partial r}{\partial z_{m}} & \frac{\partial r}{\partial v_{m z}} \\
\frac{\partial \theta_{r}}{\partial x_{m}} & \frac{\partial \theta_{r}}{\partial v_{m x}} & \frac{\partial \theta_{r}}{\partial y_{m}} & \frac{\partial \theta_{r}}{\partial v_{m y}} & \frac{\partial \theta_{r}}{\partial z_{m}} & \frac{\partial \theta_{r}}{\partial v_{m z}} \\
\frac{\partial \varphi_{r}}{\partial x_{m}} & \frac{\partial \varphi_{r}}{\partial v_{m x}} & \frac{\partial \varphi_{r}}{\partial y_{m}} & \frac{\partial \varphi_{r}}{\partial v_{m y}} & \frac{\partial \varphi_{r}}{\partial z_{m}} & \frac{\partial \varphi_{r}}{\partial v_{m z}}
\end{array}\right], \\
& \frac{\partial r}{\partial x_{m}}=-\frac{x_{f}-x_{m}}{\sqrt{\left(x_{f}-x_{m}\right)^{2}+\left(y_{f}-y_{m}\right)^{2}+\left(z_{f}-z_{m}\right)^{2}}}, \\
& \frac{\partial r}{\partial y_{m}}=-\frac{y_{f}-y_{m}}{\sqrt{\left(x_{f}-x_{m}\right)^{2}+\left(y_{f}-y_{m}\right)^{2}+\left(z_{f}-z_{m}\right)^{2}}} \\
& \frac{\partial r}{\partial z_{m}}=-\frac{z_{f}-z_{m}}{\sqrt{\left(x_{f}-x_{m}\right)^{2}+\left(y_{f}-y_{m}\right)^{2}+\left(z_{f}-z_{m}\right)^{2}}} \\
& \frac{\partial r}{\partial v_{m x}}=\frac{\partial r}{\partial v_{m y}}=\frac{\partial r}{\partial v_{m z}}=0, \\
& \frac{\partial \theta_{r}}{\partial x_{m}}=\frac{\left(y_{f}-y_{m}\right)\left(x_{f}-x_{m}\right)}{\sqrt{\left(x_{f}-x_{m}\right)^{2}+\left(z_{f}-z_{m}\right)^{2}} \cdot\left[\left(x_{f}-x_{m}\right)^{2}+\left(y_{f}-y_{m}\right)^{2}+\left(z_{f}-z_{m}\right)^{2}\right]}, \\
& \frac{\partial \theta_{r}}{\partial y_{m}}=-\frac{\left(x_{f}-x_{m}\right)^{2}+\left(z_{f}-z_{m}\right)^{2}}{\sqrt{\left(x_{f}-x_{m}\right)^{2}+\left(z_{f}-z_{m}\right)^{2}} \cdot\left[\left(x_{f}-x_{m}\right)^{2}+\left(y_{f}-y_{m}\right)^{2}+\left(z_{f}-z_{m}\right)^{2}\right]}, \\
& \frac{\partial \theta_{r}}{\partial z_{m}}=\frac{\left(y_{f}-y_{m}\right)\left(z_{f}-z_{m}\right)}{\sqrt{\left(x_{f}-x_{m}\right)^{2}+\left(z_{f}-z_{m}\right)^{2}} \cdot\left[\left(x_{f}-x_{m}\right)^{2}+\left(y_{f}-y_{m}\right)^{2}+\left(z_{f}-z_{m}\right)^{2}\right]}, \\
& \frac{\partial \theta_{r}}{\partial v_{m x}}=\frac{\partial \theta_{r}}{\partial v_{m y}}=\frac{\partial \theta_{r}}{\partial v_{m z}}=0, \\
& \frac{\partial \varphi_{r}}{\partial x_{m}}=\frac{z_{f}-z_{m}}{\left(x_{f}-x_{m}\right)^{2}+\left(z_{f}-z_{m}\right)^{2}}, \\
& \frac{\partial \varphi_{r}}{\partial z_{m}}=\frac{x_{f}-x_{m}}{\left(x_{f}-x_{m}\right)^{2}+\left(z_{f}-z_{m}\right)^{2}}, \\
& \frac{\partial q_{z}}{\partial v_{m x}}=\frac{\partial q_{z}}{\partial v_{m y}}=\frac{\partial q_{z}}{\partial v_{m z}}=\frac{\partial q_{z}}{\partial y_{m}}=0 .
\end{aligned}
$$


$K_{\theta} K_{\varphi} \varphi_{m} \theta_{m} \dot{\varphi}_{r}$ and $\dot{\theta}_{r}$ should be estimated on the basis of the prior estimated information because they are unknown variables in state transport matrix:

$$
\begin{aligned}
& \widehat{\theta}_{r}(k)=\arccos \left(\frac{\hat{y}_{m}(k-1)-y_{f}(k-1)}{\sqrt{\left(\widehat{x}_{m}(k-1)-x_{f}(k-1)\right)^{2}+\left(\hat{y}_{m}(k-1)-y_{f}(k-1)\right)^{2}+\left(\widehat{z}_{m}(k-1)-z_{f}(k-1)\right)^{2}}}\right), \\
& \widehat{\varphi}_{r}(k)=\arcsin \left(\frac{\widehat{z}_{m}(k-1)-\widehat{z}_{f}(k-1)}{\sqrt{\left(\widehat{x}_{m}(k-1)-x_{f}(k-1)\right)^{2}+\left(\widehat{z}_{m}(k-1)-z_{f}(k-1)\right)^{2}}}\right), \\
& \widehat{\theta}_{m}(k)=\arccos \left(\frac{\widehat{v}_{m z}(k-1)}{\sqrt{\hat{v}_{m x}^{2}(k-1)+\widehat{v}_{m y}^{2}(k-1)+\widehat{v}_{m z}^{2}(k-1)}}\right), \\
& \widehat{\varphi}_{m}(k)=\arcsin \left(\frac{\widehat{\hat{v}}_{r}(k)}{\sqrt{\widehat{v}_{m x}^{2}(k-1)+\widehat{v}_{m z}^{2}(k-1)}}\right), \\
& \hat{\dot{\varphi}}_{r}(k)=\frac{\widehat{\varphi}_{r}(k)-\widehat{\theta}_{r}(k-1)}{T}, \\
& \hat{\dot{\theta}}_{m}(k)=\frac{\widehat{\theta}_{m}(k)-\widehat{\theta}_{m}(k-1)}{T}, \\
& \widehat{\dot{\varphi}}_{m}(k)=\frac{\widehat{\varphi}_{m}(k)-\widehat{\varphi}_{m}(k-1)}{T}, \\
& \widehat{K}_{\theta}(k)=\frac{\hat{\dot{\theta}}_{m}(k)}{\hat{\dot{\theta}}_{r}(k)}, \\
& \hat{\dot{\varphi}}_{m}(k) \\
& \hat{\dot{\varphi}}_{r}(k)
\end{aligned}
$$

The EKF with the PN motion model to track air-to-air missile is shown in Algorithm 1.

\subsection{The Particle Filter}

3.2.1. The Sequential Importance Sampling (SIS) Algorithm. The Particle Filter algorithm to deal with the nonlinear tracking problem has been used on a wide consensus [6]. Let $\mathbf{Z}_{1: k}=\{\mathbf{Z}(1), \mathbf{Z}(2), \ldots, \mathbf{Z}(k)\}$ denote a sequence of observations and $p\left(\mathbf{X}(k) \mid \mathbf{Z}_{1: k}\right)$ denote the posterior density of $\mathbf{X}(k)$. We can acquire Bayesian estimation $\widehat{\mathbf{X}}(k)$ and $\widehat{\mathbf{P}}(k)$ by

$$
\begin{aligned}
& \widehat{\mathbf{X}}(k)=\int \mathbf{X}(k) p\left(\mathbf{X}(k) \mid \mathbf{Z}_{1: k}\right) d \mathbf{X}(k), \\
& \widehat{\mathbf{P}}(k)=\int(\mathbf{X}(k)-\widehat{\mathbf{X}}(k))(\mathbf{X}(k)-\widehat{\mathbf{X}}(k))^{\mathrm{T}} \\
& \cdot p\left(\mathbf{X}(k) \mid \mathbf{Z}_{1: k}\right) d \mathbf{X}(k) .
\end{aligned}
$$

The calculation of the posterior density requires integrals, but the normalizing integral cannot be evaluated analytically and numerical integration over possibly high-dimensional spaces is infeasible. The ideal of SIS algorithm is that the required posterior density function is represented by a set of random samples with associated weight $\left\{\mathbf{x}^{(i)}(k), \omega^{(i)}(k)\right\}_{i=1}^{N_{s}}$ and to compute state estimates in the basis of these samples and weights. According to SIS algorithm, the posterior density $p\left(\mathbf{X}(k) \mid \mathbf{Z}_{1: k}\right)$ can be approximated as

$$
p\left(\mathbf{X}(k) \mid \mathbf{Z}_{1: k}\right) \approx \sum_{i=1}^{N_{s}} \omega^{(i)}(k) \delta\left(\mathbf{X}(k)-\mathbf{x}^{(i)}(k)\right),
$$

where the weights $\omega^{(i)}(k)$ are defined in

$$
\begin{aligned}
& \omega^{(i)}(k) \propto \omega^{(i)}(k-1) \\
& . \frac{p\left(\mathbf{Z}(k) \mid \mathbf{x}^{(i)}(k)\right) p\left(\mathbf{x}^{(i)}(k) \mid \mathbf{x}^{(i)}(k-1)\right)}{q\left(\mathbf{x}^{(i)}(k) \mid \mathbf{x}^{(i)}(k-1), \mathbf{Z}(k)\right)},
\end{aligned}
$$




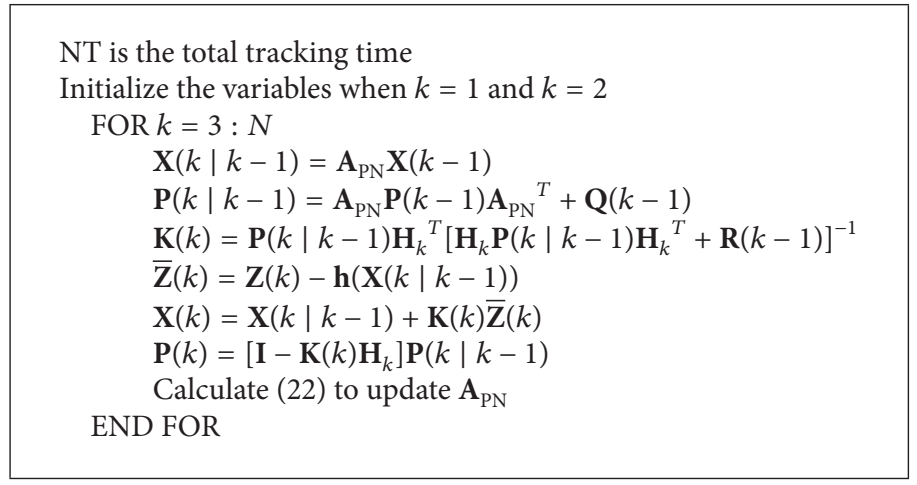

Algorithm 1

where $q\left(\mathbf{x}^{(i)}(k) \mid \mathbf{x}^{(i)}(k-1), \mathbf{Z}(k)\right)$ is termed the importance sampling proposal distribution. It is easy to draw samples from the distribution defined by $q\left(\mathbf{x}^{(i)}(k) \mid \mathbf{x}^{(i)}(k-1), \mathbf{Z}(k)\right)$, but it is hard to draw samples directly from the posterior density $p\left(\mathbf{X}(k) \mid \mathbf{Z}_{1: k}\right)$. In standard Particle Filter, we set $q\left(\mathbf{x}^{(i)}(k) \mid \mathbf{x}^{(i)}(k-1), \mathbf{Z}(k)\right)$ as $p\left(\mathbf{x}^{(i)}(k) \mid \mathbf{x}^{(i)}(k-1)\right)$ :

$$
\begin{array}{r}
q\left(\mathbf{x}^{(i)}(k) \mid \mathbf{x}^{(i)}(k-1), \mathbf{Z}(k)\right) \\
\quad=p\left(\mathbf{x}^{(i)}(k) \mid \mathbf{x}^{(i)}(k-1)\right) .
\end{array}
$$

Then

$$
\omega^{(i)}(k) \propto \omega^{(i)}(k-1) p\left(\mathbf{Z}(k) \mid \mathbf{x}^{(i)}(k)\right) .
$$

Assuming that $\mathbf{v}(k)$ and $\mathbf{w}(k)$ are mutually independent zero-mean white Gaussian processes [7] in (1) and (5), the prior density $p\left(\mathbf{x}^{(i)}(k) \mid \mathbf{x}^{(i)}(k-1)\right)$ and the likelihood density $p\left(\mathbf{Z}(k) \mid \mathbf{x}^{(i)}(k)\right)$ become

$$
\begin{aligned}
& p\left(\mathbf{x}^{(i)}(k) \mid \mathbf{x}^{(i)}(k-1)\right) \\
& \quad=N\left(\mathbf{x}^{(i)}(k) ; \mathbf{A}_{\mathrm{PN}} \mathbf{x}^{(i)}(k-1), \mathbf{Q}(k)\right), \\
& p\left(\mathbf{Z}(k) \mid \mathbf{x}^{(i)}(k)\right)=N\left(\mathbf{Z}(k) ; \mathbf{h}\left(\mathbf{x}^{(i)}(k)\right), \mathbf{R}(k)\right) .
\end{aligned}
$$

3.2.2. The Resampling Step. After a few iterations with SIS, the variance of the importance weights increases because most particles will have negligible weights. This is called particle degeneracy. Fixing on an importance sampling proposal distribution, one way to mitigate the particle degeneracy is to adopt the resampling step. The effective sample size $N_{\text {eff }}$ denotes an index variable of degeneracy introduced in [6]. $N_{\text {eff }}$ can be estimated approximately by

$$
N_{\mathrm{eff}} \approx \frac{1}{\sum_{i=1}^{N_{s}}\left(\widetilde{\omega}^{(i)}(k)\right)^{2}} .
$$

If $N_{\text {eff }}$ falls below a threshold $N_{T}$, the resampling algorithm will sample from (24) to generate a new set $\left\{\mathbf{x}^{(i) *}(k)\right\}_{i=1}^{N_{s}}$ and reset the weights $\omega^{(i) *}(k)$ to $1 / N_{s}$.

The standard PF with the PN motion model to track the air-to-air missile is shown in Algorithm 2.
NT is the total tracking time

Initialize the variables when $k=1$ and $k=2$

FOR $k=3: N$

$$
\begin{aligned}
& \text { FOR } i=1: N_{s} \\
& \text { Draw } \mathbf{x}^{(i)}(k) \sim p\left(\mathbf{x}^{(i)}(k) \mid \mathbf{x}^{(i)}(k-1)\right) \\
& \text { Assign the particle a weight according to (27) } \\
& \text { END FOR } \\
& \text { FOR } j=1: N_{s} \\
& \text { Normalize the weights by } \\
& \widetilde{\omega}^{(j)}(k)=\omega^{(j)}(k) / \sum_{i=1}^{N_{s}}\left(\omega^{(i)}(k)\right)^{2} \\
& \text { END FOR } \\
& \text { Estimate } \widehat{\mathbf{X}}(k) \text { and } \widehat{\mathbf{P}}(k) \text { by } \\
& \widehat{\mathbf{X}}(k)=\sum_{j=1}^{N_{s}} \widetilde{\omega}^{(j)}(k) \mathbf{x}^{(j)}(k) \\
& \widehat{\mathbf{P}}(k)=\sum_{j=1}^{N_{s}} \widetilde{\omega}^{(j)}(k)\left(\mathbf{x}^{(j)}(k)-\widehat{\mathbf{X}}(k)\right)\left(\mathbf{x}^{(j)}(k)-\widehat{\mathbf{X}}(k)\right)^{\mathrm{T}} \\
& \text { Calculate }(22) \text { to update } \mathbf{A}_{\mathrm{PN}} \\
& \text { Calculate } N_{\text {eff }} \text { using }(29) \\
& \text { IF } N_{\text {eff }}<N_{T} \\
& \text { Take resampling algorithm }
\end{aligned}
$$$$
\text { IF } N_{\text {eff }}<N_{T}
$$$$
\text { End FOR }
$$

\section{Algorithm 2}

3.3. The Genetic Algorithm Particle Filter. The standard Particle Filter can solve the particle degeneracy, but the resampling algorithm relying on the high weight particles leads to the loss diversity [7]. The evolution idea of Genetic Algorithm (GA) [8] can be adopted in the resampling step to solve the problem. The chromosomes and adaptability in the GA are corresponding to the particle and weight in the PF, respectively. So we can use the selection, intercross, and variation steps to deal with the particle in resampling step to increase the diversity.

GA for the resampling step is shown in Algorithm 3.

The GAPF uses the GA to replace the standard resampling step in the PN and increases the diversity, which is validated by simulations.

\section{Simulation Results}

\subsection{Hardware Condition}

An Intel ${ }^{\circledR} \mathrm{Core}^{\mathrm{TM}} \mathrm{i} 3-2100 @ 3.10 \mathrm{GHz}$ CPU is used with 2.00 GB RAM and MATLAB 2010b. 


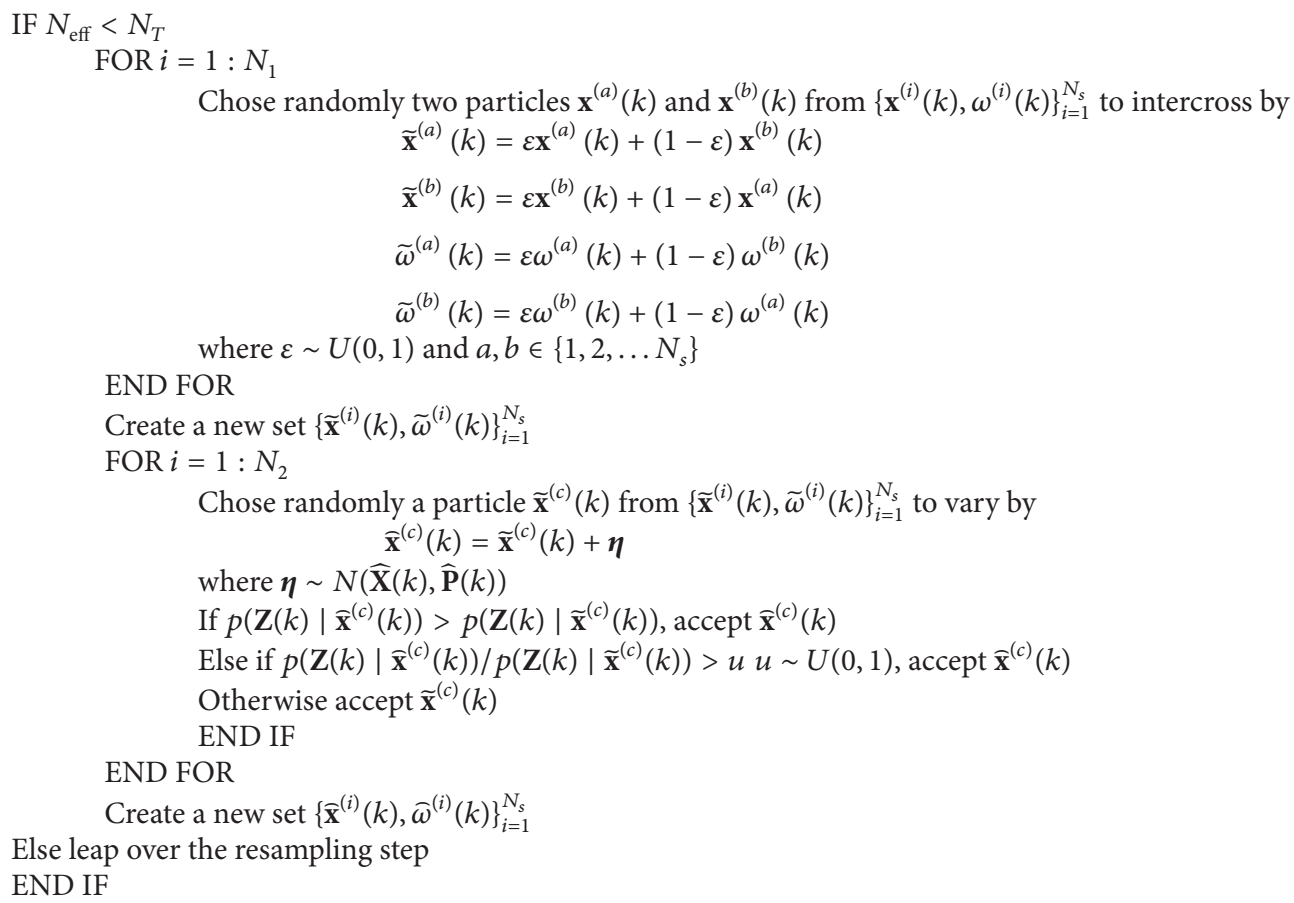

Algorithm 3

4.2. The True Ballistic Trajectory of the Air-to-Air Missile. We assume that the aircraft attacked flies along a straight line at a constant velocity $\left|v_{f}\right|=200 \mathrm{~m} / \mathrm{s}$ with the pitching angle $\theta_{f}=$ $\pi / 120$ and the azimuth angle $\varphi_{f}=\pi / 6$ at the original point $(10000,500,10000)$ in north-sky-west geographic coordinate system. We take the firing point of the air-to-air missile as the coordinate origin $(0,0,0)$ and assume that the missile moves following the Proportional Navigation law, whose proportional parameter $K_{\theta}=K_{\varphi}=4$, at a constant speed $v_{m}=500 \mathrm{~m} / \mathrm{s}$ with the pitching angle $\theta_{m}=0$ and the azimuth angle $\varphi_{m}=0$. Through the missile dynamics equation set (refer to [3]), we can acquire the ballistic trajectory of the missile and the measurement set $\mathbf{Z}$ with a second sampling interval in Figure 2.

4.3. The Simulation Results. In Figure 3, we show the missile state estimates filtered by the $\mathrm{CV}, \mathrm{CA}$, and $\mathrm{PN}$ models with EKF, respectively. In $\mathrm{CV}$ model, we assume that the original state is $\mathbf{X}(k=1)=(0,0,0,0,0,0)$; the estimate covariance matrix is $\mathbf{P}(k=1)=\operatorname{diag}(100,100,100,30$, $30,30)$; the state noise covariance matrix is $\mathbf{Q}=\operatorname{diag}(0,0$, $0,50,50,50)$; the measurement noise covariance matrix is $\mathbf{R}=\operatorname{diag}(200,0.04,0.04)$. In CA model, we assume that the original state and covariance matrix are $\mathbf{X}(k=1)=(0,0$, $0,0,0,0,0,0,0)$ and $\mathbf{P}(k=1)=\operatorname{diag}(100,100,100,30,30,30$, $10,10,10)$ separately; the state noise covariance matrix is $\mathbf{Q}=\operatorname{diag}(0,0,0,0,0,0,30,30,30)$; the measurement noise covariance matrix is $\mathbf{R}=\operatorname{diag}(200,0.04,0.04)$. In PN model, we assume that the proportional parameters is $K_{\theta}(k=1)=$ $K_{\theta}(k=2)=3$ and $K_{\varphi}(k=1)=k(k=2)=2.5$; the angles

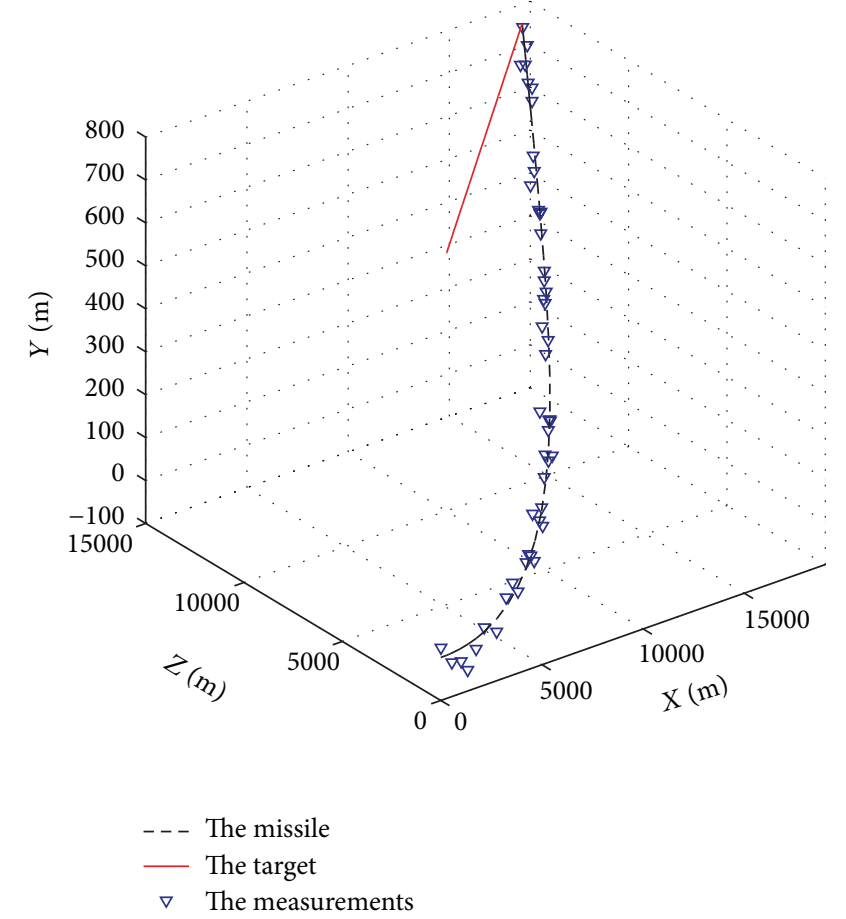

FIGURE 2: The true ballistic trajectory and the measurements.

are initialized by the measurement vectors at $k=1$ and $k=2$. Figure 3 shows that the three models are all convergent, but the state estimate performances are different among them. 


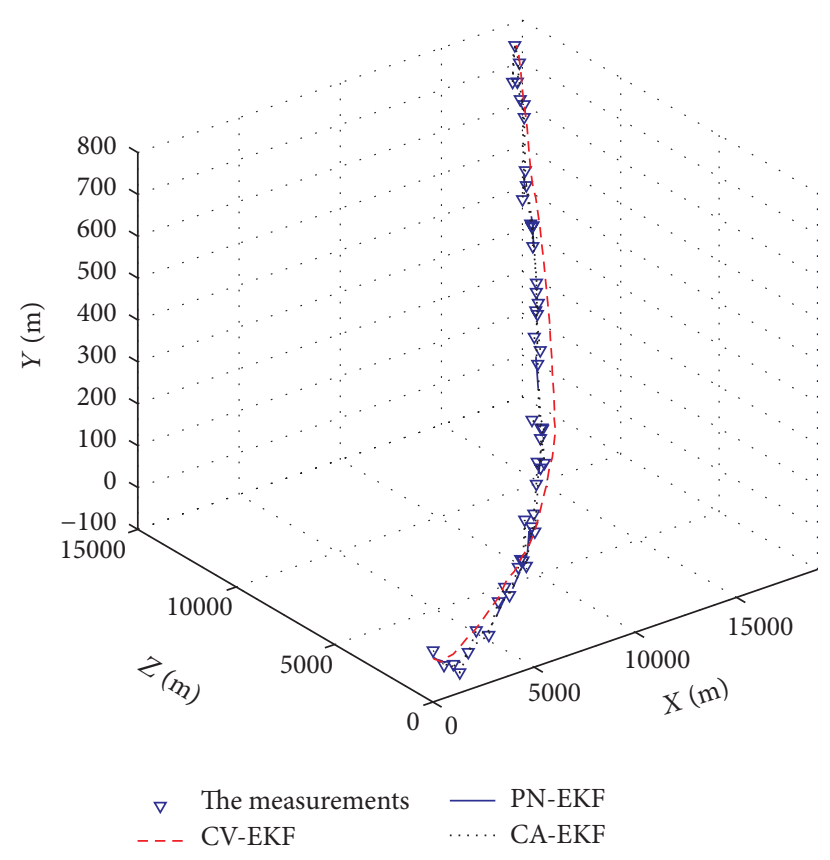

FIgure 3: The EKF track with the CV CA and PN.

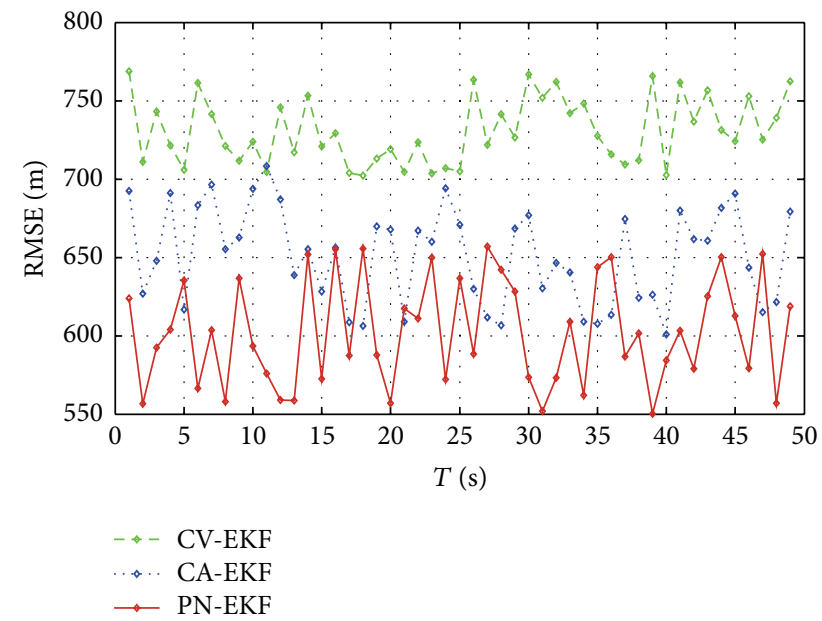

FIgure 4: The RMSE with the CV CA and PN.

Using 100 times Monte Carlo simulations for every model with EKF, the estimate effects are given in the form of the position Mean Square Error (RMSE) [21]. In Figure 4, we record the RMSE according to the models with EKF, for models comparison purposes. In Figure 5, we show that the average estimated values of proportional parameters $K_{\theta}$ and $K_{\varphi}$ are close to the true values gradually. The plot shows that PN is the best model among them for tracking the air-to-air missile.

In Figure 6, we adopt three filter algorithms, such as EKF, $\mathrm{PF}$, and GAPF, to track the missile with the constant PN model. The PN-EKF algorithm is a comparison which is the same in Figures 3 and 4 . The number of particles is $N_{s}=$ 3000 and the resampling threshold is $N_{T}=1000$ in the

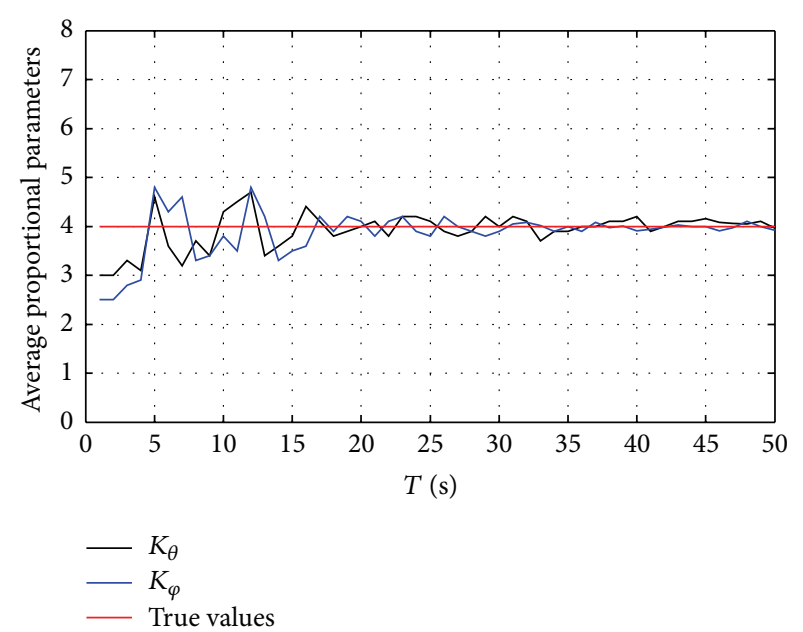

FIgURE 5: Average proportional parameters.

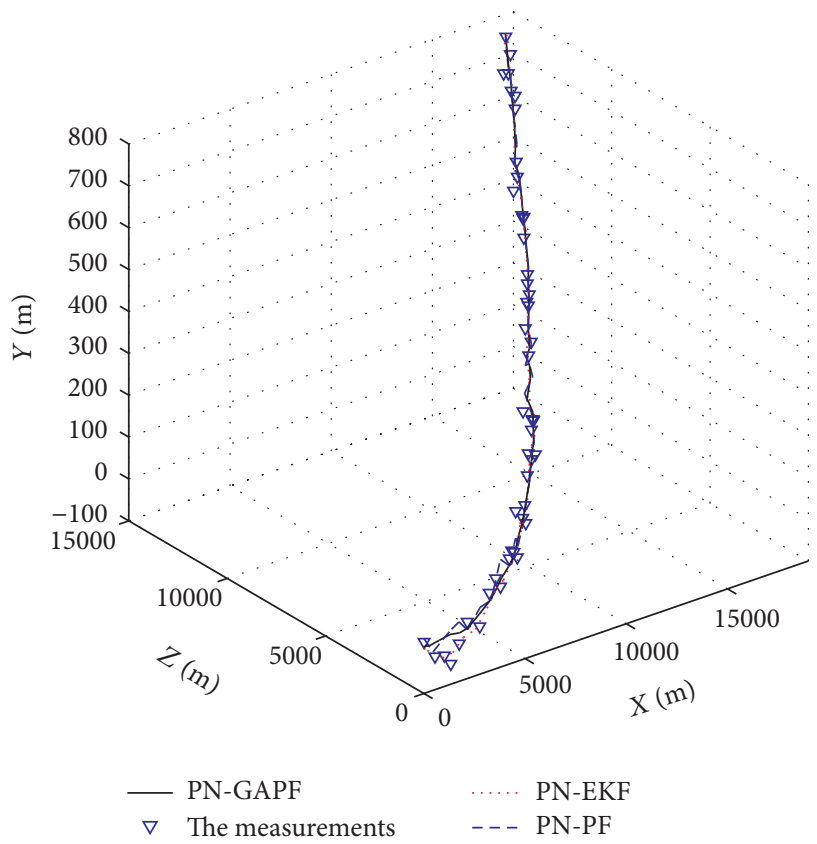

FIgURE 6: The track with PN-EKF PN-PF and PN-GAPF.

PN-PF and PN-GAPF. The number of intercross particles is $N_{1}=0.3 N_{s}$ and the number of variation particle is $N_{2}=$ $0.005 N_{s}$ in the PN-GAPF. Figure 6 shows that the GAPF performance is better than EKF and PF. The RSME curves in Figure 7 directly prove it. In Figure 8, we show that the average computation time of per step in the EKF is shortest among them, but GAPF needs more time in intercross and variation steps than the standard resampling algorithm in the PF. Further improvements in performance can be acquired by increasing the number of particles, but the computation time also becomes longer, which heavily leads to not meeting realtime tracking requirement. 


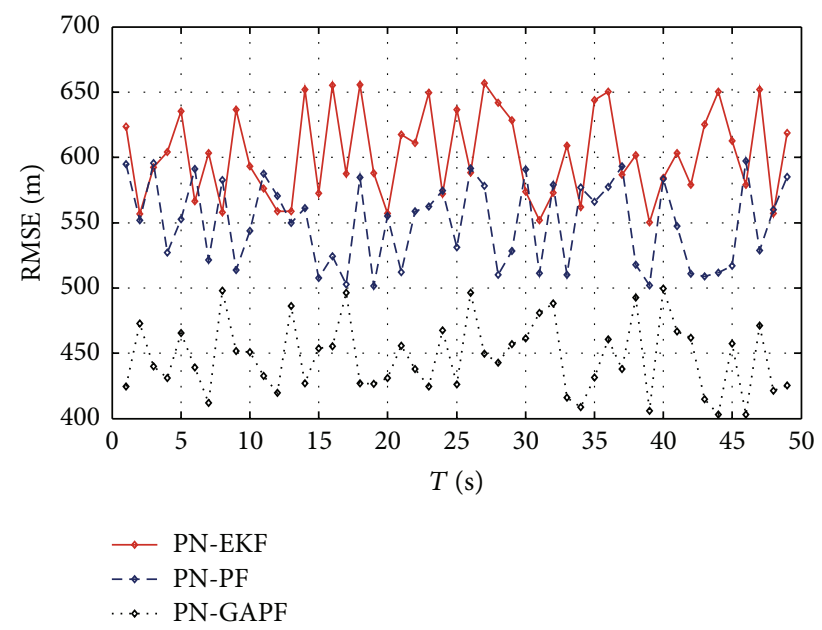

FIgURE 7: The track RMSE with PN-EKF PN-PF and PN-GAPF.

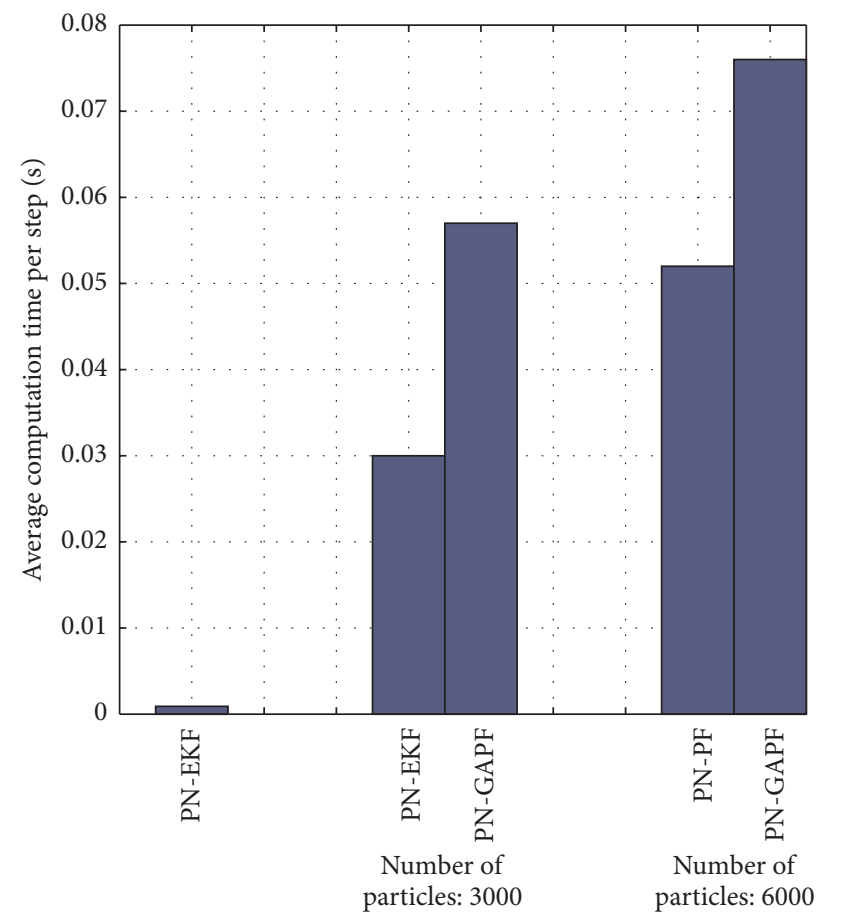

FIGURE 8: Average computation time of per step.

\section{Conclusion}

This paper aims to solve the problem of tracking the airto-air missile. Firstly we establish the PN motion model based on the PN law to preferably track the air-to-air missile in 3d Cartesian coordinate system. Secondly, the nonlinear filter problem should be solved because of the nonlinear measurement equation in the state space model for tracking the missile. In the nonlinear filter algorithms, we take the standard EKF and PF to track the missile with the PN motion model. However, both filters' results are not good because the linear approximate degree is inadequate for EKF and the diversity of particles is deficient for the standard resampling step. We introduce the intercross and variation steps in the GA to the resampling step in the standard PF for increasing the diversity. The simulation results show that the GAPN, which replaces the standard resampling step with the GA, can obtain a better performance for tracking the missile with $\mathrm{PN}$. Meanwhile, the PN model proposed in this paper is a more suitable model for the air-to-air missile than $\mathrm{CV}$ and CA models.

\section{Notations}

A:

$a_{x}(k)$ :

$a_{y}(k)$ :

$a_{z}(k)$ :

$\mathbf{h}(\cdot)$ :

$\mathbf{H}_{k}$ :

$K_{\theta}$

$K_{\varphi}:$

$N_{\text {eff }}:$

$N_{t}$ :

$T:$

$\mathbf{P}(k \mid k-1)$ :

$\mathbf{P}(k-1)$ :

$p(\cdot \mid \cdot)$ :

$q(\cdot):$

$\mathbf{Q}(k)$ :

$\mathbf{R}(k)$ :

$r(k)$ :

$v_{x}(k)$ :

$v_{y}(k):$

$v_{z}(k)$ :

$\mathbf{X}(k)$ :

$x(k)$ :

$y(k)$ :

$z(k)$ :

$\mathbf{Z}(k)$ : $\left\{\mathbf{x}^{(i)}(k), \omega^{(i)}(k)\right\}_{i=1}^{N_{s}}$ :

$\theta(k)$ :

$\varphi(k)$ :

$\mathbf{w}(k)$ :

$\mathbf{v}(k)$ :
The state transition matrix

The accelerator on $x$-axis

The accelerator on $y$-axis

The accelerator on $z$-axis

The nonlinear measurement function

The first-order Taylor expansion of the nonlinear function $\mathbf{h}(\cdot)$

The Proportional Navigation coefficient in the "OXY" plane

The Proportional Navigation coefficient in the "OXZ" plane

The effective sample size

The true sample size

The sampling period

The prediction state covariance matrix

The estimation state covariance matrix

The conditional probability

The importance sampling proposal distribution

The process noise covariance matrix

The measurement noise covariance matrix

The distance between aircraft attacked and air-to-air missile

The velocity on $x$-axis

The velocity on $y$-axis

The velocity on $z$-axis

The state vector

The position on $x$-axis

The position on $y$-axis

The position on $z$-axis

The measurement vector

: The set of random samples $\mathbf{x}^{(i)}(k)$ with associated weight $\omega^{(i)}(k)$

The pitching angle

The azimuth angle

The measurement noise

The process noise.

\section{Competing Interests}

The authors declare that they have no competing interests. 


\section{References}

[1] J. Karelahti, K. Virtanen, and T. Raivio, "Near-optimal missile avoidance trajectories via receding horizon control," Journal of Guidance, Control, and Dynamics, vol. 30, no. 5, pp. 1287-1298, 2007.

[2] F. Imado and T. Kuroda, "Engagement tactics for two missiles against an optimally maneuvering aircraft," Journal of Guidance, Control, and Dynamics, vol. 34, no. 2, pp. 574-582, 2011.

[3] G. Carpenter and M. Falco, Analysis of Aircraft Evasion Strategies in Air-to-air Missile Effectiveness Models, Grumman Aerospace Corporation, 1975.

[4] S. Y. Ong and B. L. Pierson, "Optimal planar evasive aircraft maneuvers against proportional navigation missiles," Journal of Guidance, Control, and Dynamics, vol. 19, no. 6, pp. 1210-1215, 1996.

[5] I. Moir, A. G. Seabridge, and M. Jukes, Military Avionics Systems, Thomson Press, New Delhi, India, 2005.

[6] M. Sanjeev Arulampalam, S. Maskell, N. Gordon, and T. Clapp, "A tutorial on particle filters for online nonlinear/non-Gaussian Bayesian Tracking," IEEE Transactions on Signal Processing, vol. 50, no. 2, pp. 174-188, 2002.

[7] Y. Bar-Shalom, P. Willett, and X. Tian, Tracking and Data Fusion: A Handbook of Algorithms, YBS Publishing, Storrs, Conn, USA, 2011.

[8] J. H. Holland, “Genetic algorithm," Scientific American, vol. 34, no. 9, pp. 44-50, 1992.

[9] F. Campillo and V. Rossi, "Convolution particle filter for parameter estimation in general state-space models," IEEE Transactions on Aerospace and Electronic Systems, vol. 45, no. 3, pp. 1063-1072, 2009.

[10] N. Bergman, A. Doucet, and N. Gordon, "Optimal estimation and Cramér-Rao bounds for partial non-Gaussian state space models," Annals of the Institute of Statistical Mathematics, vol. 53, no. 1, pp. 97-112, 2001.

[11] X. R. Li and V. P. Jilkov, "Survey of maneuvering target tracking. Part I. Dynamic models," IEEE Transactions on Aerospace and Electronic Systems, vol. 39, no. 4, pp. 1333-1364, 2003.

[12] T. L. Song, "Observability of target tracking with range-only measurements," IEEE Journal of Oceanic Engineering, vol. 24, no. 3, pp. 383-387, 1999.

[13] L. Lin, T. Kirubarajan, and Y. Bar-Shalom, "Pursuer identification and time-to-go estimation using passive measurements from an evader," IEEE Transactions on Aerospace and Electronic Systems, vol. 41, no. 1, pp. 190-204, 2005.

[14] N. J. Gordon, D. Salmond, and C. Ewing, "Bayesian state estimation for tracking and guidance using the bootstrap filter," Journal of Guidance, Control, and Dynamics, vol. 18, no. 6, pp. 1434-1443, 1995.

[15] F. Imado and S. Miwa, "Missile guidance algorithm against high- g barrel roll maneuvers," Journal of Guidance, Control, and Dynamics, vol. 17, no. 1, pp. 123-128, 1994.

[16] Y. Oshman, J. Shinar, and S. A. Weizman, "Using a multiplemodel adaptive estimator in a random evasion missile/aircraft encounter," Journal of Guidance, Control, and Dynamics, vol. 24, no. 6, pp. 1176-1186, 2001.

[17] T. Shima, Y. Oshman, and J. Shinar, "Efficient multiple model adaptive estimation in ballistic missile interception scenarios," Journal of Guidance, Control, and Dynamics, vol. 25, no. 4, pp. 667-675, 2002.
[18] V. Shaferman and T. Shima, "Cooperative multiple-model adaptive guidance for an aircraft defending missile," Journal of Guidance, Control, and Dynamics, vol. 33, no. 6, pp. 1801-1813, 2010.

[19] J. Shinar and D. Steinberg, "Analysis of optimal evasive maneuvers based on a linearized two-dimensional kinematic model," Journal of Aircraft, vol. 14, no. 8, pp. 795-802, 1977.

[20] B. Jung, K.-S. Kim, and Y. Kim, "Guidance law for evasive aircraft maneuvers using artificial intelligence," in Proceedings of the AIAA Guidance, Navigation, and Control Conference and Exhibit, Austin, Tex, USA, August 2003.

[21] T. Kirubarajan, Y. Bar-Shalom, K. R. Pattipati, and I. Kadar, "Ground target tracking with variable structure IMM estimator," IEEE Transactions on Aerospace and Electronic Systems, vol. 36, no. 1, pp. 26-46, 2000.

[22] Z. Wang, X. Liu, Y. Liu, J. Liang, and V. Vinciotti, "An extended kalman filtering approach to modeling nonlinear dynamic gene regulatory networks via short gene expression time series," IEEE/ACM Transactions on Computational Biology and Bioinformatics, vol. 6, no. 3, pp. 410-419, 2009. 


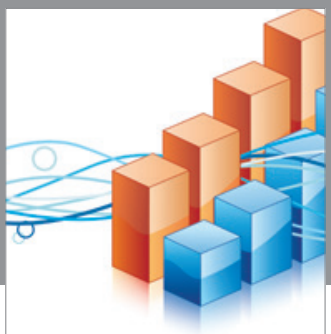

Advances in

Operations Research

vatem alat4

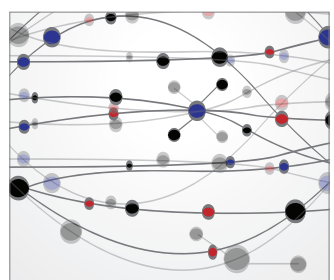

\section{The Scientific} World Journal
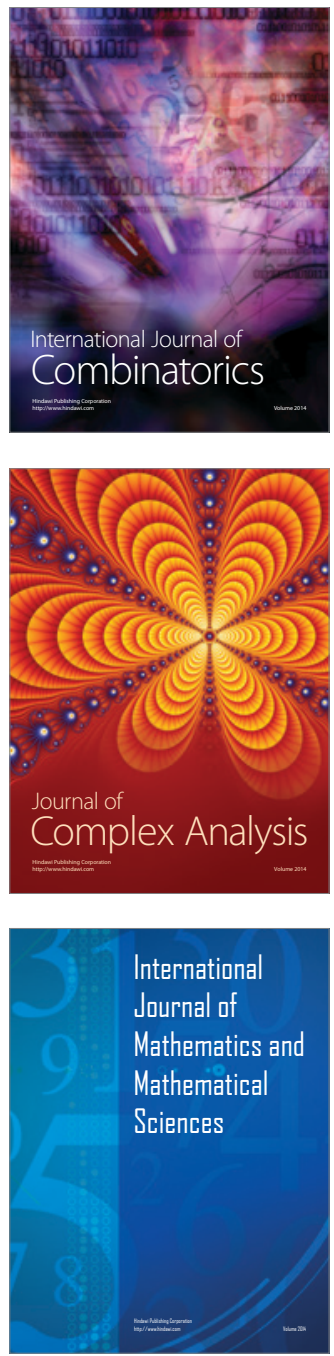
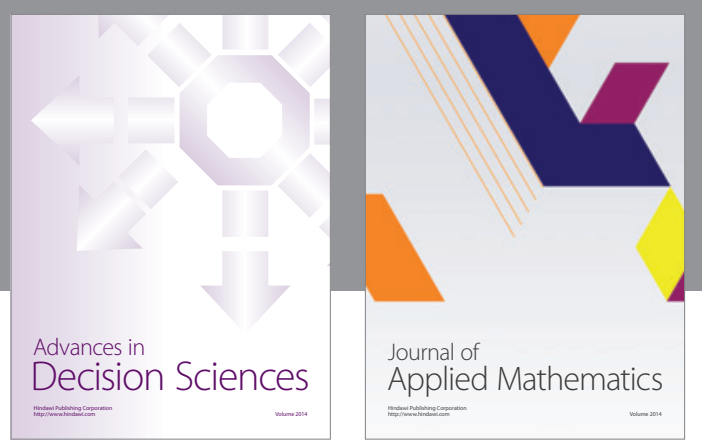

Algebra

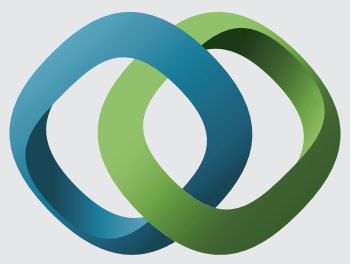

\section{Hindawi}

Submit your manuscripts at

http://www.hindawi.com
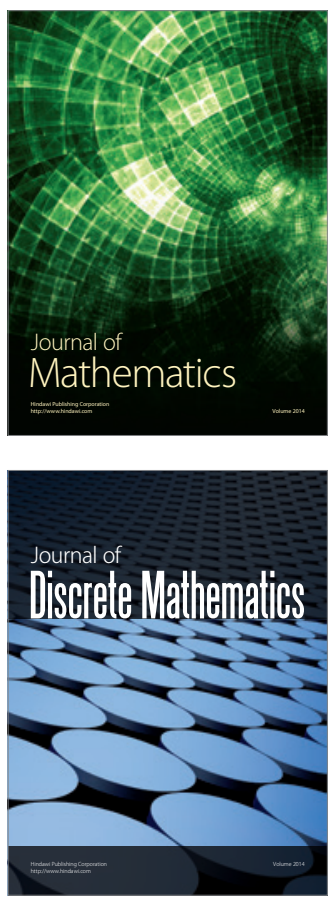

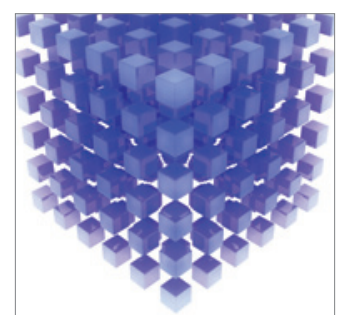

Mathematical Problems in Engineering
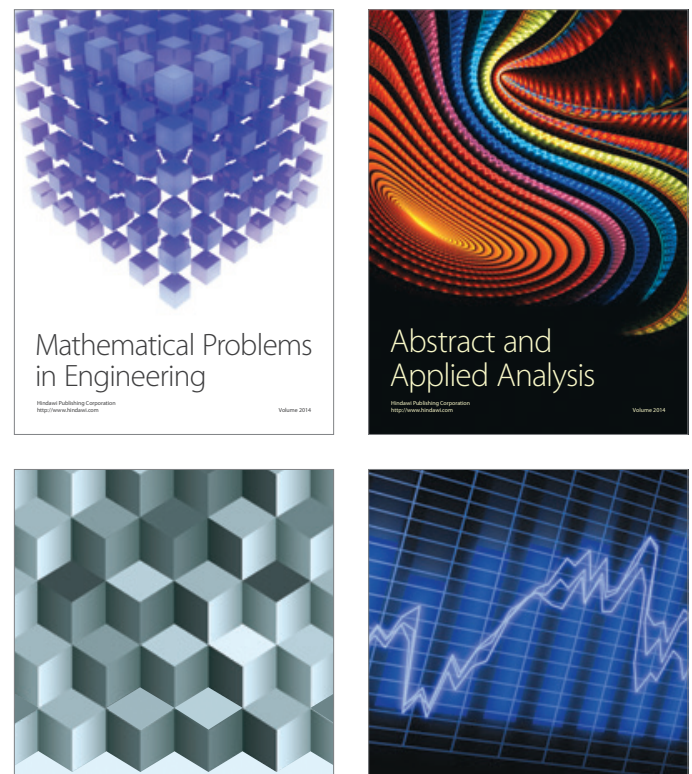

Journal of

Function Spaces

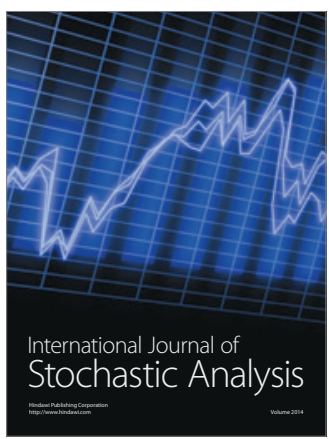

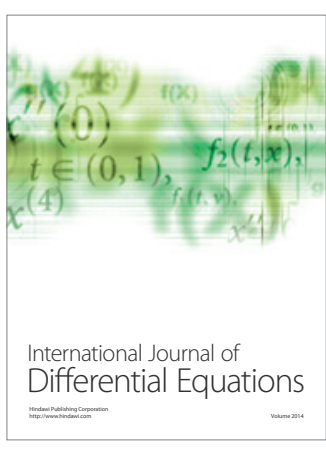
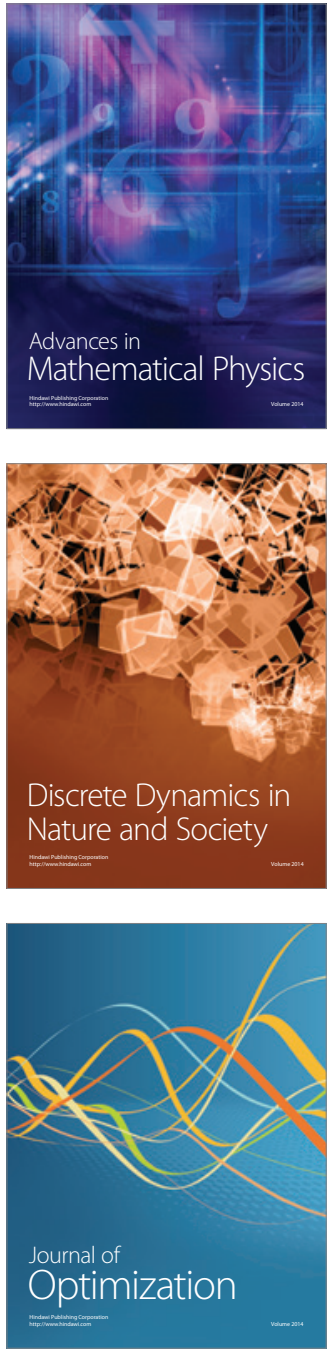